\title{
Local extraocular extension of retinoblastoma following intraocular surgery
}

\author{
K E STEVENSON,' J HUNGERFORD,' AND A GARNER ${ }^{2}$ \\ From ${ }^{1}$ Moorfields Eye Hospital, City Road, London EC1V 2PD, and the ${ }^{2}$ Institute of Ophthalmology, \\ Cayton Street, London EC1V 7AT
}

SUMMARY Three cases of local extraocular extension of retinoblastoma following intraocular surgery via a transscleral approach have been seen in one centre during a 12-month period. This number represents a significant increase on the previous incidence of this serious complication, which may be related to more widespread use of vitrectomy techniques. Orbital and local lymph node recurrence of retinoblastoma have been associated with a very poor survival rate in the past, and steps must be taken to limit the chance of this happening should an intraocular surgical procedure be performed inadvertently on an eye containing a retinoblastoma. Early enucleation without implant is advised, and adjuvant orbital radiotherapy is strongly recommended, even when there is no histopathological evidence of local extraocular tumour spread.

Retinoblastoma is the most common primary intraocular tumour of childhood, with an incidence of between 1 in 14000 and 1 in 34000 live births.' The average age at diagnosis is 18 months. Bilateral cases usually present earlier, at a mean age of 12 months but rarely present after age 5 years. Unilateral cases usually present at a mean age of 23 months but may occur well into the teens. ${ }^{2}$

Retinoblastoma presenting late is often misdiagnosed as intraocular inflammation. Misdiagnosis may lead to intraocular surgery in an attempt to confirm an inflammatory or other cause or in an attempt to improve vision. This results in a delay in establishing the correct diagnosis, which, in allowing tumour growth to continue unabated, predisposes to extraocular spread either by direct extrascleral extension or via the severed end of the optic nerve. ${ }^{3}$

Intraocular surgical procedures have a more direct and serious adverse effect on survival rate in retinoblastoma, because they predispose to local extraocular spread by breaching the natural barrier provided by the sclera or cornea. Extraocular spread alone is an adverse prognostic factor, but when it leads to orbital recurrence or local lymph node metastasis the mortality rate is extremely high. ${ }^{4}$

We report three cases of retinoblastoma in older children referred to a specialist centre for Correspondence to Mr J Hungerford, FRCS, Moorfields Eye Hospital, City Road, London EC1V 2PD. management of orbital recurrence or local lymph node metastasis. In all three the diagnosis of retinoblastoma had not been suspected initially but had been made on the results of intraocular biopsy.

\section{Case reports}

CASE I

An 8-year-old girl was referred for treatment of a mass in her enucleated right socket. ${ }^{5}$ She had initially presented in April 1986, then aged 7, with a onemonth history of a red right eye and decreased vision. She was found to have cells in the anterior chamber, iris nodules, vitreous opacities, and retinal exudates with a secondary glaucoma. The left eye was normal. A presumptive diagnosis of panophthalmitis had been made and the initial treatment was with topical steroids and acetazolamide, to which she showed no response.

In July 1986, four months after presentation, she underwent a right pars plana lensectomy in an attempt to improve her vision. During surgery she was noted to have an area of white friable tissue overlying the inferior pars plana. Cells from this area were aspirated and found to be highly suspicious of retinoblastoma. Three weeks later the right eye was enucleated and an Allen orbital implant inserted.

Histological examination of the globe revealed 739 


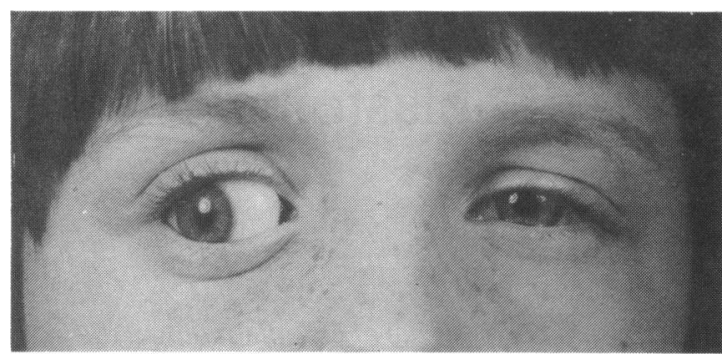

Fig. 1 Tilting of the ocular prosthesis in case 1 .

to the anterior segment in the form of diffuse infiltration of the iris and ciliary body. Seeding of the anterior vitreous was also present, but the retinal component of the tumour was confined to the anterior periphery. The severed end of the optic nerve was not affected, and there was no choroidal invasion. She received no further treatment postoperatively.

Six months after enucleation she presented with tilting and forward displacement of her right prosthesis with a rapid increase in orbital swelling and pain, and was referred for specialist management (Figs. 1, 2). High resolution CT scanning showed enhancing tissue in the right orbit but no evidence of intracranial tumour. Cytological study of material obtained by bone marrow aspiration and trephine showed no evidence of malignant infiltration by standard histological techniques, but a few cells were positive to tesing with a monoclonal antibody against neurectoderm (UJ13A). Cytological examination of the cerebrospinal fluid (CSF) showed no evidence of disseminated retinoblastoma.

In March 1987 an excision biopsy of the right orbital mass was performed, revealing undifferentiated retinoblastoma extending to the margins of resection. A biopsy of the posterior orbit was free of tumour. Review of the histology of the enucleated eye at this stage showed a few tumour cells in the sclera in a haemorrhagic area presumed to correspond to the initial biopsy site. The patient then received radiotherapy to the right orbit, $4400 \mathrm{cGy} \mathrm{(44}$ Gy) in 22 fractions over 30 days, and six courses of chemotherapy with vincristine, cyclophosphamide, cis-platinum, and etoposide, with intrathecal methotrexate. She remained free of disease 21 months after her orbital recurrence.

\section{CASE 2}

A 5-year-old girl was referred for management of a preauricular lymph node metastasis of retinoblastoma following enucleation of her right eye three months earlier. She had presented initially, two weeks after minor trauma to her right eye in September 1985, with decreased vision, a right divergent squint, and

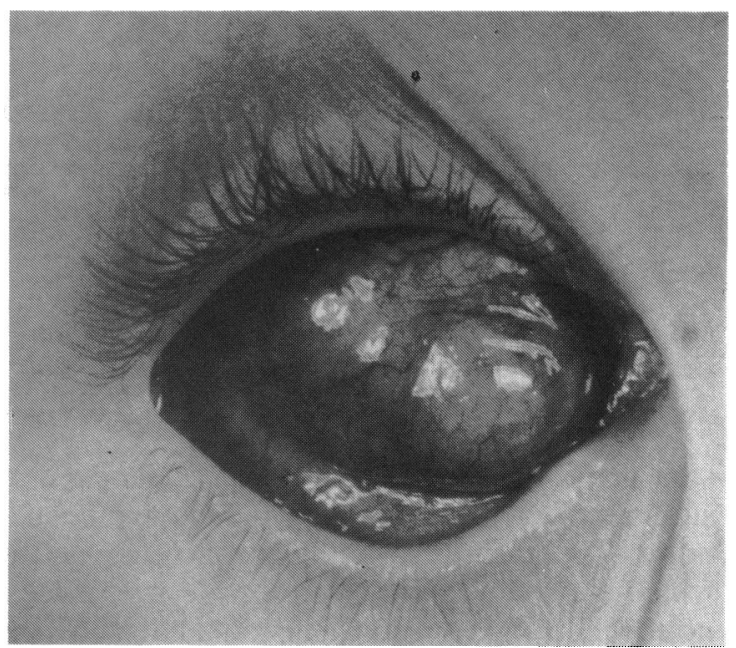

Fig. 2 Orbital recurrence of retinoblastoma in case 1.

heterochromia. She was noted to have rubeosis iridis, and some white, solid-looking lumps associated with vitreous haemorrhage were seen behind a clear crystalline lens. A CT scan showed no evidence of an intraocular foreign body or mass lesion. A B scan ultrasound of the right eye showed a very dense vitreous opacity with no evidence of a solid mass. No mention was made of any intraocular calcification. One month later a right vitreous biopsy was performed.

Histological examination of the specimen showed clumps of cells with basophilic nuclei which were thought to be malignant. In December 1985 she underwent a right enucleation and acrylic ball implant. Histological examination of the enucleated globe showed retinoblastoma infiltrating the optic nerve, though the cut end of the nerve was free of tumour. There was no substantial choroidal invasion by the tumour, and no comment was made on the appearance of the sclerostomy site. No further treatment had been given after enucleation.

Three months later she presented again with an enlarged right preauricular node and was referred for further treatment. A lymph node biopsy showed retinoblastoma, though no tumour mass could be demonstrated in the right orbit by CT scan. Systemic staging investigations including bone marrow aspiration and trephine and cytological examination of the CSF were negative for disseminated retinoblastoma. She was treated with radiotherapy to the right parotid region to a total dose of $4000 \mathrm{cGy}$ in 20 fractions over 28 days. Microscopic residual orbital tumour was presumed to be present, and the right orbit was included in the treatment field. Radiotherapy was followed by six courses of chemotherapy 


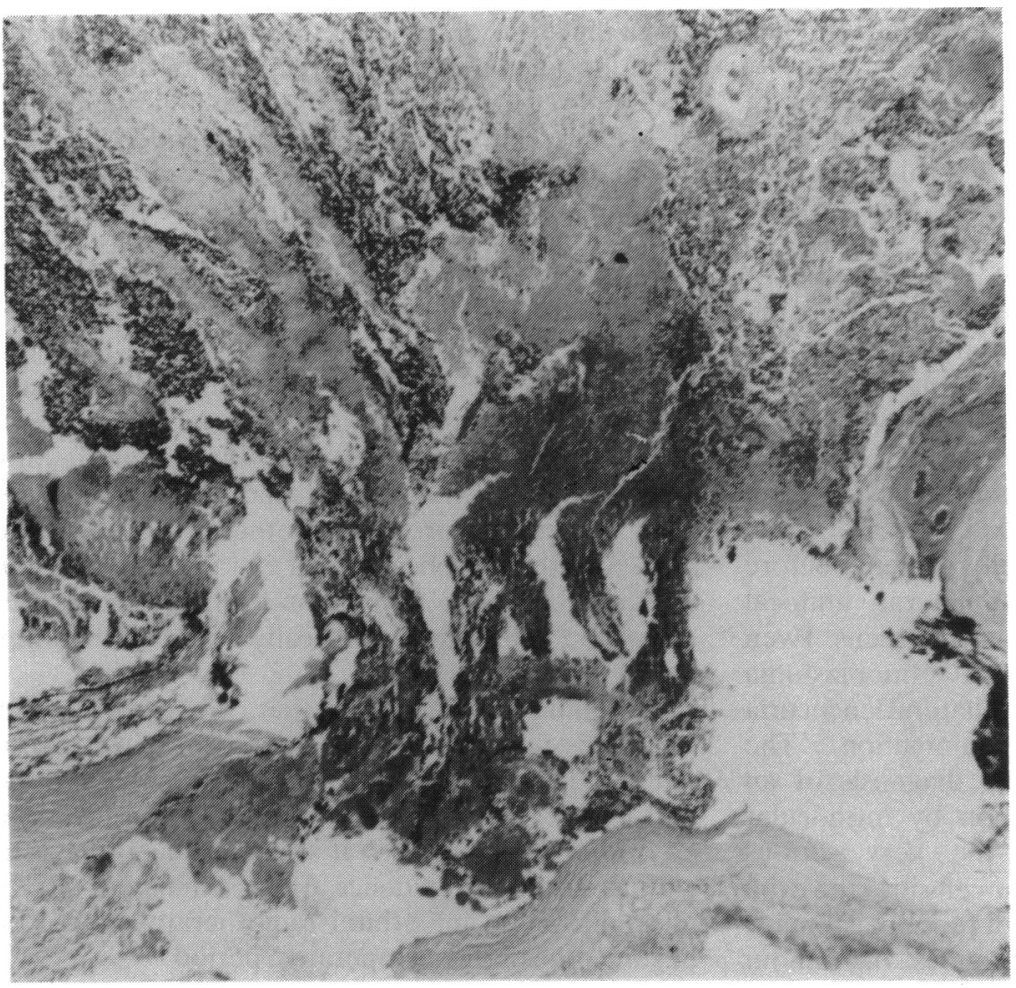

Fig. 3 Section of the sclerostomy site in case 3 to show prolapsing necrotic tumour tissue. (H and $E, \times 22)$.

as in the first case. She remained free of tumour 18 months later.

\section{CASE 3}

This 6-year-old girl was referred for management of orbital recurrence of retinoblastoma. She had presented initially with leucocoria in the right eye. A tentative diagnosis of Coats's disease had been made, but she subsequently underwent a biopsy which was performed beneath a partial thickness scleral flap. The histological diagnosis proved to be retinoblastoma. Four days after the biopsy she underwent a right enucleation. Histological examination of the enucleated eye showed a poorly differentiated retinoblastoma with choroidal invasion. There was extension of the tumour into the optic nerve head, no comment being made about the severed end of the nerve. There was a large gaping scleral perforation through which necrotic tumour tissue had prolapsed (Fig. 3). No further treatment had been given after enucleation.

In October 1986, three months later, she presented with an orbital recurrence of the tumour and was referred for specialist management. Excision biopsy of the orbital mass was positive for retinoblastoma. Systemic staging investigations were negative for disseminated retinoblastoma, and she underwent radiotherapy to the right socket, receiving $4500 \mathrm{cGy}$ in 23 fractions over 37 days, and six courses of chemotherapy as detailed previously. She remained free of disease 11 months later.

\section{Discussion}

Three cases of orbital or local lymph node metastasis of retinoblastoma presenting to a specialist centre during a 12-month period are described. An intraocular operation had been performed in all three cases prior to the establishment of the diagnosis of retinoblastoma, and none of the cases had received any specific treatment directed towards reducing the likelihood of subsequent orbital recurrence.

Orbital or local lymph node recurrence of retinoblastoma is uncommon. In one large series from the same specialist centre only 16 cases were reported among 317 children referred during a 15 -year period between 1970 and $1984 .{ }^{4}$ Only one of these 16 had undergone intraocular surgery prior to the diagnosis of retinoblastoma being established. The present group of patients is consecutive with that previously reported and represents a substantial increase in the number of cases with intraocular procedures performed prior to enucleation. This increase from one case in 16 years to three cases within one year is 
alarming and may be related to new and more widely available technology in vitreous surgery.

Orbital or lymph node recurrence of retinoblastoma is a very serious event. Only one of the 16 cases previously reported from this centre is a long-term survivor. ${ }^{4}$ This child was free of disseminated retinoblastoma on systemic staging and was treated with a combination of excision biopsy of an orbital recurrence, radical orbital radiotherapy, and systemic adjuvant chemotherapy. This was the index case on which the management plan chosen for the three children herein described was based. Although all three patients have sustained clinical remissions following this treatment, they cannot yet be counted long-term survivors.

Although retinoblastoma usually presents before the age of 5 years, sporadic, unilateral, unifocal retinoblastoma may occur well into the teens. Even in the older child the diagnosis of retinoblastoma must be considered in any case of unilateral leucocoria with signs of intraocular inflammation. The advantages of achieving a tissue diagnosis of an intraocular inflammatory condition by intraocular biopsy must be set against the very serious consequences of releasing tumour cells into the orbit if the diagnosis proves to be one of retinoblastoma.

Rodriguez $^{6}$ has reported examples of tumour seeding along 19-gauge needle tracks at three and six weeks after biopsy. He suggests that extraocular extension of the tumour is more likely to occur if enucleation is delayed or if the entry site of the instrument is immediately beneath the bulk of the tumour. Transcorneal aqueous aspiration has been used for many years as an aid to the diagnosis of retinoblastoma with anterior segment involvement. ${ }^{\text {? }}$ The method carries the theoretical advantages that the entry site is not subconjunctival, so that it can be easily observed for signs of tumour recurrence and deep orbital seeding of the tumour is unlikely. Nevertheless superficial conjunctival implantation of tumour cells may occur, and any affected conjunctiva might not be removed during enucleation. The risk of extraocular seeding of retinoblastoma following a breach of scleral or corneal barrier has long been recognised. 'It is questionable if it is legitimate to aspirate the subretinal fluid for diagnostic purposes since any escape into the orbit may lead to extraocular spread; and it is certainly illegitimate to puncture the vitreous, a procedure which has led to rapid fungation into the orbit." 8
If a diagnosis of retinoblastoma is made as a result of an intraocular biopsy, vitrectomy, or lensectomy, strenuous efforts should be made to limit the chance of orbital recurrence. It is recommended that the affected eye should be enucleated as soon as possible and subjected to detailed histological examination, with particular attention being paid to the sclerostomy sites. The enucleated socket will require frequent examination, and to facilitate this an orbital implant should not be inserted. Of eight patients in whom histological examination of the enucleated eye revealed evidence of local extraocular spread of retinoblastoma and who received orbital radiotherapy soon after enucleation, none had recurrence in the orbit. ${ }^{4}$ Adjuvant orbital radiotherapy is therefore advised in the presence of local extraocular extension and may be a wise precaution even if tumour cells cannot be detected histologically at the sclerostomy site.

In summary, we advise that, when intraocular surgery is inadvertently performed on an eye containing a retinoblastoma, enucleation should be performed as soon as possible, without insertion of an orbital implant. Even if systemic staging investigations show no evidence of disseminated retinoblastoma, adjuvant orbital radiotherapy should be begun in the early postoperative period.

Dr J Harry kindly provided histological slides of case 3 for further study. We thank Mr C M P Claoue for helpful discussion, and Walter for secretarial help.

\section{References}

1 Tarkkanen A, Tuovinen E. Retinoblastoma in Finland 19121964. Acta Ophthalmol (Kbh) 1971; 49: 293-300.

2 Shields JA. Diagnosis and management of intraocular tumors. St Louis: Mosby, 1983.

3 Stafford WR, Yanoff M, Parnell BL. Retinoblastomas initially misdiagnosed as primary intraocular inflammations. Arch Ophthalmol 1969; 82: 771-3.

4 Hungerford J, Kingston J, Plowman N. Orbital recurrence of retinoblastoma. Ophthalmic Paediatr Genet 1987; 8: 63-8.

5 Garner A, Kanksi JJ, Kinnear F. Retinoblastoma: report of a case with minimal retinal involvement but massive anterior segment spread. Br J Ophthalmol 1987; 71: 858-63.

6 Rodriguez A. Diagnosis of retinoblastoma by cytologic examination of the aqueous and the vitreous. Mod Probl Ophthalmol 1977; 18: 142-8.

7 Reese AB. Tumors of the eye. 2nd ed. Philadelphia: Harper and Row, 1963: 111-2.

8 Duke-Elder S. Chapter VI Cysts and tumours of the retina. In: Duke-Elder S, Dobree JH, eds. A system of ophthalmology. Volume X, London: Kimpton, 1967: 10: chapter 6: 711.

Accepted for publication 24 February 1989. 